\title{
In Utero Exposure to Norbuprenorphine, a Major Metabolite of Buprenorphine, Induces Fetal Opioid Dependence and Leads to Neonatal Opioid Withdrawal Syndrome ${ }^{\mathbb{S}}$
}

\author{
Bryce A. Griffin, Caitlin O. Caperton, Lauren N. Russell, Christian V. Cabanlong, \\ Catheryn D. Wilson, Kyle R. Urquhart, Bradford S. Martins, Marcelle Dina Zita, \\ Amy L. Patton, Alexander W. Alund, S. Michael Owens, William E. Fantegrossi, \\ Jeffery H. Moran, and Lisa K. Brents \\ Department of Chemistry and Biochemistry, University of Arkansas, Fayetteville, Arkansas (B.A.G.); Departments of \\ Pharmacology and Toxicology (C.O.C., L.N.R., C.V.C., C.D.W., K.R.U., S.M.O., W.E.F., J.H.M., L.K.B.), Psychiatry (B.S.M.), and \\ Microbiology and Immunology (M.D.Z.), University of Arkansas for Medical Sciences, Little Rock, Arkansas; and PinPoint \\ Testing, LLC, Little Rock, Arkansas (A.L.P., A.W.A., J.H.M.)
}

Received December 4, 2018; accepted April 25, 2019

\begin{abstract}
Buprenorphine is the preferred treatment of opioid use disorder during pregnancy but can cause fetal opioid dependence and neonatal opioid withdrawal syndrome (NOWS). Notably, withdrawal severity is independent of maternal buprenorphine dose, suggesting that interindividual variance in pharmacokinetics may influence risk and severity of NOWS. Using a rat model of NOWS, we tested the hypothesis that clinically relevant doses of the active metabolite norbuprenorphine (NorBUP) can induce in utero opioid dependence, manifested as naltrexone-precipitated withdrawal signs in the neonate. Pregnant Long-Evans rats were implanted with 14-day osmotic minipumps containing vehicle, morphine (positive control), or NorBUP (0.3-10 mg/kg per day) on gestation day 9 . By 12 hours post-delivery, an intraperitoneal injection of the opioid antagonist naltrexone (1 or $10 \mathrm{mg} / \mathrm{kg}$ ) or saline was
\end{abstract}

administered to pups. Precipitated withdrawal signs were graded by raters blinded to treatment conditions. In a separate group, NorBUP concentrations in maternal and fetal blood and brain on gestation day 20 were determined by liquid chromatography-tandem mass spectrometry. Steady-state maternal blood concentrations of NorBUP in dams infused with 1 or $3 \mathrm{mg} / \mathrm{kg}$ per day were comparable to values reported in pregnant humans treated with buprenorphine (1.0 and $9.6 \mathrm{ng} / \mathrm{ml}$, respectively), suggesting a clinically relevant dosing regimen. At these doses, NorBUP increased withdrawal severity in the neonate as shown by an evaluation of 10 withdrawal indicators. These findings support the possibility that NorBUP contributes to fetal opioid dependence and NOWS following maternal buprenorphine treatment during pregnancy.

\section{Introduction}

Neonatal opioid withdrawal syndrome (NOWS) is the opioid-specific type of neonatal abstinence syndrome (NAS), a potentially life-threatening condition exhibited by newborns

The project described was supported by the Arkansas Biosciences Institute Tobacco Settlement Funds, the ASPET Summer Undergraduate Research Fellowship, UAMS Child Health Initiative Award, and the UAMS Translational Research Institute (TRI) through the National Institutes of Health National Center for Advancing Translational Sciences [1U54TR001629-01A1 and KL2TR000063]. Certain aspects of the analytical work were supported in part by the National Institutes of Health National Institute on Drug Abuse [R01DA039143]. The content is solely the responsibility of the authors and does not necessarily represent the official views of the funding agencies. The authors have no conflicts of interest related to this manuscript.

Abstracts that included portions of the work presented in this manuscript were presented at the 2018 Association of Clinical and Translational Science Meeting, April 19-21, Washington DC, and the 80th Annual Scientific Meeting (2018) College on Problems of Drug Dependence, June 8-15, San Diego, CA. https://doi.org/10.1124/jpet.118.254219.

S This article has supplemental material available at jpet.aspetjournals.org. who were born physically dependent on a drug. NOWS develops within days after delivery in the absence of opioid exposure and is characterized by extreme irritability, high-pitched inconsolable crying, tremor, vomiting, diarrhea, hypersensitivity to stimuli, hyperalgesia, and sleep disturbances (Jansson et al., 2009). Because of increased opioid abuse during pregnancy, NAS diagnoses in the United States have increased more than 5-fold since 2000 (Patrick et al., 2012, 2015), and healthcare costs associated with treating children who have NAS now exceed $\$ 1.5$ billion per year (Patrick et al., 2015).

Maternal opioid use disorder (OUD) during pregnancy is a major cause of chronic in utero opioid exposure and subsequent NOWS. NOWS also is a potential side effect of buprenorphine, a partial agonist at the $\mu$-opioid receptor that is used in the medication-assisted treatment of OUD. Buprenorphine decreases maternal illicit drug use (Brogly et al., 2014), improves birth outcomes (Zedler et al., 2016), and decreases neonatal withdrawal severity (Jones et al., 2010) relative to methadone,

ABBREVIATIONS: ANOVA, analysis of variance; DMSO, dimethyl sulfoxide; GD, gestation day; LC-MS-MS, liquid chromatography-tandem mass spectrometry; NAS, neonatal abstinence syndrome; NorBUP, norbuprenorphine; NorBUP-D3, deuterated norbuprenorphine; NOWS, neonatal opioid withdrawal syndrome; OUD, opioid use disorder; PEG-400, polyethylene glycol-400. 
a full-agonist opioid that also is used to treat OUD. Despite its reduced NOWS liability, approximately $50 \%$ of neonates born to women treated with buprenorphine during pregnancy require pharmacological treatment of NOWS (Jones et al., 2010; Bartu et al., 2012). Notably, neonatal withdrawal severity is not related to maternal buprenorphine dose (Jones et al., 2014; O'Connor et al., 2016; Shah et al., 2016). This clinical observation suggests that fetal exposure to buprenorphine or to its major, active metabolite norbuprenorphine (NorBUP) may be influenced by differences in maternal-fetal metabolism, distribution, and/or excretion of buprenorphine and NorBUP among maternal-fetal dyads. Thus, defining the physiologic mechanisms that influence fetal exposure to buprenorphine and NorBUP could enable the development of improved OUD treatments exhibiting lower NOWS liability.

The role of NorBUP in buprenorphine-associated NOWS is poorly defined; however, NorBUP is a high-affinity full agonist of $\mu$ - and $\delta$-opioid receptors (Huang et al., 2001), suggesting that it may induce physical dependence following chronic exposure. Importantly, NorBUP concentrations in human umbilical cord blood, which is part of the fetal blood supply, were directly correlated to neonatal withdrawal severity in newborns following chronic prenatal treatment with buprenorphine. In contrast, cord blood concentrations of buprenorphine and buprenorphine-glucuronide, another major metabolite of buprenorphine, were uncorrelated and inversely correlated, respectively, with neonatal withdrawal severity (Shah et al., 2016). This suggests that fetal NorBUP exposure may contribute to physical dependence and subsequent NOWS following prenatal buprenorphine treatment, but little is known about the role of NorBUP in buprenorphineassociated NOWS.

To our knowledge, the present study is the first to test the hypothesis that chronic in utero exposure to clinically relevant levels of NorBUP can induce physical dependence and lead to severe withdrawal signs shortly after delivery. To test this hypothesis, we chronically treated pregnant rats with NorBUP and tested their pups for antagonist-precipitated withdrawal signs shortly after delivery. Because male neonates may exhibit more severe NOWS than females (Jansson et al., 2007, 2010; O'Connor et al., 2013; Charles et al., 2017), we assessed the rat pups for sex differences in withdrawal severity. We also quantified the concentrations of NorBUP in maternal blood during gestation to ensure that blood levels in the rat model were comparable to those in the pregnant women undergoing buprenorphine treatment. Additionally, we assessed fetal exposure to NorBUP by quantifying NorBUP concentrations in the fetal blood and brain following prenatal treatment with NorBUP, and we related NorBUP concentrations in late-gestation fetal brain to neonatal withdrawal severity. Our results fill gaps in knowledge of the role of NorBUP in buprenorphine-associated NOWS and implicate fetal NorBUP exposure as a contributor to the risk and severity of NOWS caused by prenatal buprenorphine treatment.

\section{Materials and Methods}

Free base NorBUP was provided by the National Institute on Drug Abuse (NIDA) Drug Supply Program (Bethesda, MD). Morphine sulfate salt pentahydrate and naltrexone hydrochloride were purchased from Sigma-Aldrich (St. Louis, MO). Dimethyl sulfoxide (DMSO) and polyethylene glycol-400 (PEG-400) were purchased from Fisher Scientific.
NorBUP free base and morphine sulfate salt pentahydrate were dissolved in a vehicle consisting of 1:2:1 DMSO/PEG-400/sterile saline and loaded into osmotic minipumps (Model 2ML2; Alzet, Cupertino, CA), which were primed overnight in sterile saline at $37^{\circ} \mathrm{C}$, per manufacturer's guidelines, prior to subcutaneous implantation into pregnant rats. Naltrexone hydrochloride (Sigma-Aldrich) was dissolved in sterile saline prior to administration to pups by intraperitoneal injection. Doses of free base NorBUP, morphine sulfate salt pentahydrate, and naltrexone hydrochloride are reported as milligrams per kilogram; for brevity, we refer to the drugs as NorBUP, morphine, and naltrexone, respectively, throughout the article. ToxBox analytical plates (PinPoint Testing, LLC, Little Rock, AR) were used to quantify NorBUP in rat blood and brain. ToxBox analytical plates contained deuterated norbuprenorphine (NorBUP- $\mathrm{D}_{3}$ ) and NorBUP certified reference material that were used as internal and calibration standards, respectively.

Animal Care and Use. All studies were performed in accordance with the Guide for Care and Use of Laboratory Animals as adopted and promulgated by the National Institutes of Health. All animal protocols were approved by the Institutional Animal Care and Use Committee at the University of Arkansas for Medical Sciences.

Thirty-six timed-pregnant Long-Evans rat dams (Charles River Laboratories, Wilmington, MA) were used in the neonatal withdrawal studies. Dams arrived at the UAMS Division of Animal Laboratory Medicine animal facility on gestation day (GD) 6 ( \pm 1 day) weighing approximately $190-220$ g. Rats were singly housed in Plexiglas cages $\left(30 \times 24.6 \times 41.2 \mathrm{~cm}^{3}\right)$ with enrichment that included Environdri crinkled paper, cotton square nestlets, and tubes. Cages were individually ventilated by the Allentown NexGen Rat 900 Ecoflo system. Room temperature and humidity were maintained at $22 \pm 2{ }^{\circ} \mathrm{C}$ and $45 \%-50 \%$, respectively, on a 12/12-hour light/dark cycle in an animal facility that is accredited by the Association for Assessment and Accreditation of Laboratory Animal Care. On GD 9, osmotic minipumps containing vehicle, morphine, or NorBUP were implanted subcutaneously posterior to the scapulae, as instructed by the manufacturer while rats were maintained under isoflurane anesthesia. Subcutaneous meloxicam (1 mg/kg) was administered to rats 30-60 minutes before minipump implantation, and rats were monitored for signs of pain or infection for the first 24 hours after implantation. Dam weight gain was monitored during the study to ensure that prenatal treatment did not compromise gestation or affect dam feeding and/or fetal growth. Upon delivery on GD 22, pups were tested for opioid withdrawal (see "Precipitated Withdrawal Testing" below).

Eight additional timed-pregnant Long-Evans rats were each surgically implanted with a single jugular cannula under isoflurane anesthesia on GD 2 by the Charles River Laboratory veterinary staff prior to shipment to UAMS. The cannula exited between the scapulae, and was secured by surgical staples. Minipumps delivering 1,3 , or $10 \mathrm{mg} / \mathrm{kg}$ per day NorBUP were implanted subcutaneously on GD 9. To avoid damaging the cannula in these animals, osmotic minipump implantation was modified by implanting the minipump more posteriorly, such that the minipump was placed just above the base of the tail. Blood samples $(250 \mu \mathrm{l})$ were taken via the cannula on GDs 8 , $10,13,17$, and 20. On GD 20, fetal and maternal tissues (brain and whole blood) were harvested under inhaled isoflurane anesthesia (induced with $4 \%$; maintained with $3 \%$ ). Fetal brain and blood samples were pooled by litter. All blood samples were stored at $4^{\circ} \mathrm{C}$ in tubes containing EDTA to prevent coagulation until quantitatively analyzed using liquid chromatography-tandem mass spectrometry (LC-MS$\mathrm{MS})$. All brain samples were snap-frozen in liquid nitrogen and stored at $-80^{\circ} \mathrm{C}$ until analyzed by LC-MS-MS.

Precipitated Withdrawal Testing. Two hundred and thirtyfour neonatal rat pups were used in the neonatal withdrawal tests. Pups were removed from dams by 12 hours after delivery, counted, grouped by sex and placed in an incubator (Model 12-140; Quincy Laboratory, Inc.) maintained at $30^{\circ} \mathrm{C}$ (Zhu and Barr, 2004). Pups from each litter were randomly selected and assigned, within sex, to be challenged with an intraperitoneal injection of either saline $(10 \mathrm{ml} / \mathrm{kg})$ 
or naltrexone ( 1 or $10 \mathrm{mg} / \mathrm{kg}$ ). Pups were immediately video-recorded after injection for 10 minutes in groups of two to four. A grader, who was blinded to the treatment of the pups and their dams, rated each pup for twenty 10 -second segments that were sampled from the video every 30 seconds. The presence or absence of the following withdrawal signs was recorded: body curls, body stretches, tremor, face wiping, foreleg movement, hindleg movement, head movement, wall climbing, locomotion, and quiet behavior. These signs of neonatal opioid withdrawal were adapted from an earlier study (Jones and Barr, 2000) and are defined in Table 1. Each 10-second segment during which a pup exhibited a withdrawal sign was scored as a 1 for that sign; absence of a sign was scored as a 0 . Each pup's score for each sign was determined by summing scores for the twenty 10 -second segments, resulting in a score ranging between 0 and 20 for each sign. The scores for all signs were summed to calculate a global withdrawal score for each pup. Because quiet behavior is a negative withdrawal sign (i.e., the absence of quiet behavior indicates withdrawal), each pup's score for quiet behavior was subtracted from the maximum possible score of 20 , and this difference was summed with the pup's scores for all other signs to determine the global withdrawal score.

Quantitative Measurement of NorBUP. NorBUP was quantified in rat brain and blood using supported liquid extraction and customized, commercially available ToxBox analytical plates designed for the precise measurement of NorBUP (PinPoint Testing, LLC). Briefly, all rat brain samples, including unknown samples and untreated, blank samples for calibration and quality control (QC) material, were weighed and homogenized in five volumes $(0.2 \mathrm{~g} / \mathrm{ml})$ of $0.1 \mathrm{M}$ sodium acetate buffer ( $\mathrm{pH} 5$ ) using an Omni Bead Ruptor homogenizer (Omni Inc., NW Kennesaw, GA). Calibration standards and second-source quality control material were prepared on the ToxBox analytical plates by adding blank rat brain homogenates or blood samples $(0.1 \mathrm{~g}$ brain or $250 \mu \mathrm{l}$ whole blood) to wells containing pretitered NorBUP ranging from 1 to $50 \mathrm{ng} / \mathrm{g}$ (brain) or 0.4 to $20 \mathrm{ng} / \mathrm{ml}$ (blood) and NorBUP-D ${ }_{3}$ internal standard [1 ng/g (brain) or $0.4 \mathrm{ng} / \mathrm{ml}$ (blood)].

Unknown samples were loaded into ToxBox analytical plates containing only internal standard and processed identically to standard and quality control material. Plates were placed on a shaking incubator at $900 \mathrm{rpm}$ for approximately 15 minutes, followed by 1:1 dilution with ammonium hydroxide $(0.5 \mathrm{M})$. Plates were returned to the shaking incubator for 15 minutes at $900 \mathrm{rpm}$. Samples were then loaded onto a 96-well ISOLUTE SLE+ plate (Biotage, Charlotte, NC) and extracted using two elutions of ethyl acetate $(100 \%, 2 \times 900 \mu \mathrm{l})$ under gentle vacuum. The eluent was dried under nitrogen flow with gentle heating and reconstituted in $100 \mu \mathrm{l}$ of $100 \%$ methanol. Analysis was completed using an Agilent 1260 series quaternary liquid chromatography system that was interfaced with Agilent 6420A tandem mass spectrometer (Agilent Technologies, Santa Clara, CA). The LC-MS-MS method utilizes a Kinetex $2.6 \mu \mathrm{m}$ Phenyl-Hexyl $100 \AA$ LC Column $(50 \times$ $4.6 \mathrm{~mm}$; Phenomenex, Inc., Torrance, CA) heated to $35^{\circ} \mathrm{C}$. NorBUP and its internal standard were resolved using a $10 \mathrm{mM}$ ammonium formate/0.1\% formic acid in methanol gradient started at 95\% aqueous, ramped to $100 \%$ organic over 4 minutes, and held constant for an additional 1 minute. The gradient was then returned to initial conditions and equilibrated for an additional 2 minutes. The total run time for each analysis was 7.0 minutes, including the column equilibration period between injections. Capillary voltage and cell accelerator voltage were 2000 and $4 \mathrm{~V}$, respectively. The primary multiple reaction monitoring (MRM) transition for NorBUP was $414.3-101.1 \mathrm{~m} / \mathrm{z}$ with collision energy of $41 \mathrm{~V}$; the secondary transition was $414.3-57.3 \mathrm{~m} / z$ with collision energy of $49 \mathrm{~V}$. The fragmentor voltage for NorBUP was $216 \mathrm{~V}$. The MRM transition for the NorBUP-D ${ }_{3}$ internal standard was $417.3-101.1 \mathrm{mz}$ with collision energy of $41 \mathrm{~V}$ and fragmentor voltage of $226 \mathrm{~V}$. The limit of quantitation for NorBUP was $0.4 \mathrm{ng} / \mathrm{ml}$ in blood and $1 \mathrm{ng} / \mathrm{g}$ in brain.

Statistical Analyses. Unless otherwise stated, data are displayed as mean \pm S.E.M. All analyses were performed using GraphPad Prism, version 7.02 (GraphPad Software, La Jolla, CA).

Littermates were not considered independent observations; therefore, withdrawal sign data from littermates that received the same postnatal challenge were averaged and this average was used in analyses.

The main and interaction effects of prenatal treatment and postnatal challenge on withdrawal sign scores were evaluated using a $5 \times$ 3 factorial two-way analysis of variance (ANOVA). Prenatal treatment was evaluated at five levels: vehicle, morphine ( $15 \mathrm{mg} / \mathrm{kg}$ per day), or NorBUP $(0.3,1.0$, or $3.0 \mathrm{mg} / \mathrm{kg}$ per day); postnatal challenge was evaluated at three levels: saline or naltrexone (1 or $10 \mathrm{mg} / \mathrm{kg}$ ); resulting total was 15 groups. Main effects were examined only in the absence of an interaction effect. Significant interactions between prenatal treatment and postnatal challenge indicated opioid dependence, as naltrexone induces withdrawal signs only in opioid-dependent individuals. Dunnett's multiple comparison test was used to identify groups with elevated global withdrawal scores (relative to vehicletreated, saline-challenged pups).

The main and interaction effects of prenatal treatment and sex on global withdrawal score were assessed within each postnatal challenge group using two-way ANOVAs, and Sidak's multiple comparison test was performed to determine whether global withdrawal scores differed between males and females in any group.

To assess gestational weight gain, the weight of each dam was measured at least eight times between GD 7 and GD 21. A linear regression analysis was performed on each treatment group's data, and slopes of these regression lines were tested for statistical differences using an F-test.

The effect of dam weight on changes in maternal blood concentrations of NorBUP across gestation was assessed in groups treated with 1,3 , or $10 \mathrm{mg} / \mathrm{kg}$ per day NorBUP as follows: We determined and plotted the group mean blood concentrations of NorBUP $(y$-axis $)$ over gestation $(x$-axis) before and after correcting for weight. We corrected

TABLE 1

Behavioral definitions of opioid withdrawal signs in the neonatal rat

\begin{tabular}{|c|c|}
\hline Behavior & Definition \\
\hline Body curl & Ventral or lateral flexion of trunk \\
\hline Body stretch & $\begin{array}{l}\text { Extension or dorsal flexion of trunk causing apparent lengthening of } \\
\text { body }\end{array}$ \\
\hline Body tremor & $\begin{array}{l}\text { Mild lateral movements of head that progress to a full-body lateral } \\
\text { tremor }\end{array}$ \\
\hline Face wiping & Wiping of both forelimbs across the face \\
\hline Foreleg movements & Flexion, extension, or rotation of one or both forelegs \\
\hline Hindleg movements & Flexion or extension of one or both hindlegs \\
\hline Head movements & Ventral, dorsal or lateral rotary motion of the head \\
\hline Wall climbing & $\begin{array}{l}\text { Placing at least two forepaws on the wall of the observation cage and } \\
\text { moving them up and down }\end{array}$ \\
\hline Locomotion & Walking across the cage \\
\hline Quiet & Sedated appearance with no movement for at least $50 \%$ of the sample \\
\hline
\end{tabular}


for weight by dividing each subject's blood concentration by its weight for each gestation day. We then calculated the linear regression slopes of each group's corrected and uncorrected data and used an F-test for each treatment group to determine if the slopes of the corrected and uncorrected data significantly differed.

Repeated one-way ANOVAs with Sidak's multiple comparison test were conducted to compare NorBUP concentrations in fetal versus maternal tissues (blood and brain) on GD 20 following prenatal treatment with 1 or $3 \mathrm{mg} / \mathrm{kg}$ per day NorBUP. Maternal-fetal matching was determined to be ineffective for NorBUP concentrations in blood; therefore, the results of a one-way ANOVA with Sidak's multiple comparison test are reported for these data.

Differences in the proportion of dams in each treatment group that had spontaneous abortions were assessed using Fisher's exact test. Spontaneous abortions were signified by a reduction of maternal weight gain during prenatal treatment and no evidence of delivery up to 24 days after breeding. Litter characteristics (mean litter size, mean pup weight per litter, and mean number of each sex per litter) were assessed at the treatment group level using one-way ANOVAs.

\section{Results}

Prenatal treatment significantly interacted with postnatal challenge to affect curls, stretches, and quiet behavior (Table 2). Additionally, prenatal treatment had significant main effects on foreleg and hindleg movements, and postnatal challenge had a significant main effect on foreleg and head movements (Table 2). Scores for tremors, face wiping, locomotion, and wall climbing were not significantly affected by either factor (Supplemental Fig. 1; Table 2). As shown in Fig. 1, prenatal treatment with morphine or NorBUP ( 1 or $3 \mathrm{mg} / \mathrm{kg}$ per day) and postnatal challenge with naltrexone $(1$ or $10 \mathrm{mg} / \mathrm{kg}$ ) increased curls, stretches, and foreleg movements and decreased quiet behavior relative to prenatal treatment with vehicle and postnatal challenge with saline. Foreleg and head movements were significantly increased only in the group that was prenatally treated with $1 \mathrm{mg} / \mathrm{kg}$ per day NorBUP and postnatally challenged with $10 \mathrm{mg} / \mathrm{kg}$ naltrexone (Fig. 1).

The global withdrawal score is a summary score of all withdrawal signs. Prenatal treatment interacted with postnatal challenge to affect global withdrawal scores (Table 2). Prenatal treatment with morphine or NorBUP $(1.0$ or $3.0 \mathrm{mg} / \mathrm{kg}$ per day) and postnatal challenge with naltrexone $(1$ or $10 \mathrm{mg} / \mathrm{kg}$ ) significantly increased global withdrawal scores (relative to prenatal treatment with vehicle and postnatal challenge with saline; Fig. 2). Saline-challenged pups whose dams were treated with $3.0 \mathrm{mg} / \mathrm{kg}$ per day NorBUP also exhibited elevated global withdrawal scores (Fig. 2). There were no interactions between sex and prenatal exposure and no main effect of sex on global withdrawal scores (Supplemental Table 1). There was no difference between males and females in global withdrawal scores for any group (Fig. 3). Overall, prenatal treatment with $3 \mathrm{mg} / \mathrm{kg}$ per day NorBUP produced opioid dependence and subsequent global withdrawal severity that was comparable to $15 \mathrm{mg} / \mathrm{kg}$ per day morphine (Figs. 2 and 3).

Prenatal treatment with $10 \mathrm{mg} / \mathrm{kg}$ per day NorBUP or $20 \mathrm{mg} / \mathrm{kg}$ per day morphine was associated with high rates of spontaneous abortion, as evidenced by a reduction of maternal

TABLE 2

Two-way ANOVA results for withdrawal signs

\begin{tabular}{|c|c|c|c|c|}
\hline Sign & Predictor & $F$-Value & $P$ Value & $\%$ of Total Variation \\
\hline \multirow[t]{3}{*}{ Global } & Interaction & $F(8,50)=3.05$ & $0.0071^{a}$ & 8.721 \\
\hline & Prenatal & $\mathrm{F}(4,50)=34.8$ & $<0.0001^{a}$ & 49.74 \\
\hline & Postnatal & $\mathrm{F}(2,50)=26.8$ & $<0.0001^{a}$ & 19.14 \\
\hline \multirow[t]{3}{*}{ Curls } & Interaction & $\mathrm{F}(8,50)=2.13$ & $0.0495^{a}$ & 10.54 \\
\hline & Prenatal & $\mathrm{F}(4,50)=19.1$ & $<0.0001^{a}$ & 47.17 \\
\hline & Postnatal & $F(2,50)=8.93$ & $<0.0005^{a}$ & 11.02 \\
\hline \multirow[t]{3}{*}{ Stretches } & Interaction & $\mathrm{F}(8,50)=2.28$ & $0.0367^{a}$ & 10.03 \\
\hline & Prenatal & $\mathrm{F}(4,50)=22.8$ & $<0.0001^{a}$ & 50.30 \\
\hline & Postnatal & $F(2,50)=10.1$ & $0.0002^{a}$ & 11.15 \\
\hline \multirow[t]{3}{*}{ Tremors } & Interaction & $F(8,50)=0.45$ & 0.89 & 6.046 \\
\hline & Prenatal & $\mathrm{F}(4,50)=1.30$ & 0.28 & 8.754 \\
\hline & Postnatal & $F(2,50)=0.18$ & 0.83 & 0.6136 \\
\hline \multirow[t]{3}{*}{ Face wiping } & Interaction & NA & & \\
\hline & Prenatal & NA & & \\
\hline & Postnatal & NA & & \\
\hline \multirow[t]{3}{*}{ Foreleg Movements } & Interaction & $\mathrm{F}(8,50)=1.21$ & 0.31 & 8.011 \\
\hline & Prenatal & $\mathrm{F}(4,50)=6.11$ & $0.0004^{a}$ & 20.26 \\
\hline & Postnatal & $\mathrm{F}(2,50)=14.1$ & $<0.0001^{a}$ & 23.39 \\
\hline \multirow[t]{3}{*}{ Hindleg Movements } & Interaction & $\mathrm{F}(8,49)=1.54$ & 0.17 & 13.95 \\
\hline & Prenatal & $\mathrm{F}(4,49)=5.29$ & $0.0013^{a}$ & 23.92 \\
\hline & Postnatal & $\mathrm{F}(2,49)=1.61$ & 0.21 & 3.632 \\
\hline \multirow[t]{3}{*}{ Head Movements } & Interaction & $\mathrm{F}(8,50)=1.72$ & 0.12 & 15.41 \\
\hline & Prenatal & $F(4,50)=2.40$ & 0.06 & 10.74 \\
\hline & Postnatal & $\mathrm{F}(2,50)=5.62$ & $0.0063^{a}$ & 12.56 \\
\hline \multirow[t]{3}{*}{ Wall Climbing } & Interaction & $F(8,50)=0.52$ & 0.84 & 7.250 \\
\hline & Prenatal & $\mathrm{F}(4,50)=0.42$ & 0.79 & 2.946 \\
\hline & Postnatal & $\mathrm{F}(2,50)=0.35$ & 0.70 & 1.236 \\
\hline \multirow[t]{3}{*}{ Locomotion } & Interaction & $\mathrm{F}(8,50)=0.51$ & 0.84 & 6.638 \\
\hline & Prenatal & $\mathrm{F}(4,50)=1.35$ & 0.27 & 8.745 \\
\hline & Postnatal & $\mathrm{F}(2,50)=0.48$ & 0.62 & 1.570 \\
\hline \multirow[t]{3}{*}{ Quiet behavior } & Interaction & $\mathrm{F}(8,50)=2.22$ & $0.0417^{a}$ & 6.640 \\
\hline & Prenatal & $\mathrm{F}(4,50)=34.8$ & $<0.0001^{a}$ & 52.17 \\
\hline & Postnatal & $\mathrm{F}(2,50)=25.7$ & $<0.0001^{a}$ & 19.28 \\
\hline
\end{tabular}

${ }^{a}$ indicates statistical significance.

Prenatal, prenatal treatment factor; Postnatal, postnatal challenge factor; Interaction, interaction effect between prenatal treatment and postnatal challenge; NA, not applicable (all group means were 0). 

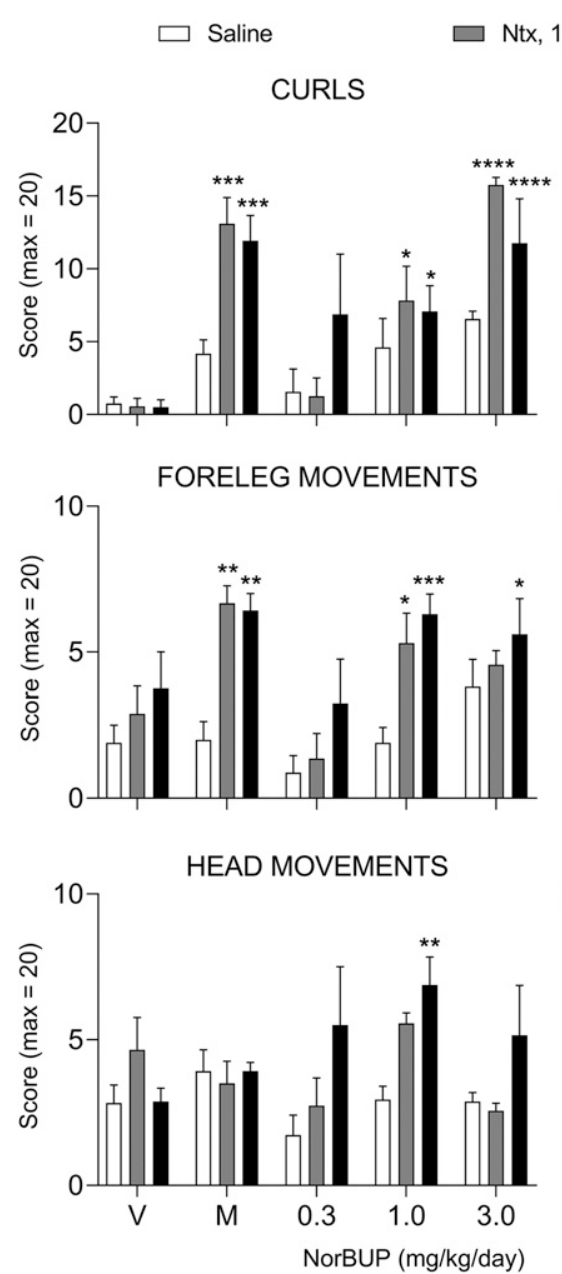

- Ntx, $10 \mathrm{mg} / \mathrm{kg}$ STRETCHES
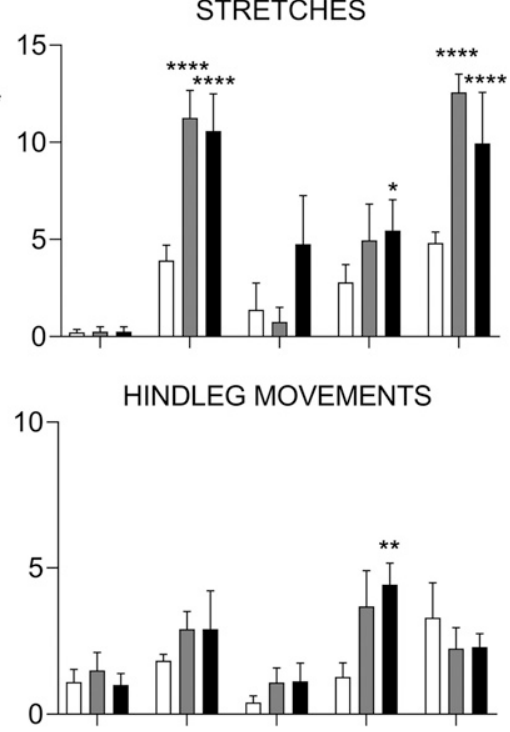

Fig. 1. Scores for neonatal withdrawal signs following prenatal treatment with NorBUP, morphine, or vehicle, and postnatal challenge with naltrexone or saline. Prenatal treatments plotted on the $x$-axis include norbuprenorphine (NorBUP, $0.3-3 \mathrm{mg} / \mathrm{kg}$ per day, s.c.), morphine (M, positive control, $15 \mathrm{mg} / \mathrm{kg}$ per day, s.c.), and vehicle (V, 1:2:1 DMSO/PEG-400/saline, $0.120 \mathrm{ml} /$ day, s.c.). Neonatal pups were challenged with saline $(10 \mathrm{ml} / \mathrm{kg}$, i.p., white bars $)$ or naltrexone (Ntx, $1 \mathrm{mg} / \mathrm{kg}$, i.p., gray bars; Ntx, $10 \mathrm{mg} / \mathrm{kg}$, i.p., black bars) on postnatal day 1 and observed for withdrawal signs. Bars represent group means of the number of 10 -second segments in which pups displayed each withdrawal sign; error bars represent S.E.M. Significant interaction or main effects of prenatal treatment and postnatal challenge were determined for the following withdrawal signs: curls, stretches, foreleg movements, hindleg movements, head movements, and quiet behavior. ${ }^{*} p<0.05$, $* * p<0.01, * * * p<0.001$, **** $p<0.0001$ vs. vehicletreated, saline-challenged pups; Dunnett's multiple comparisons test. See Table 2 for detailed statistical results.

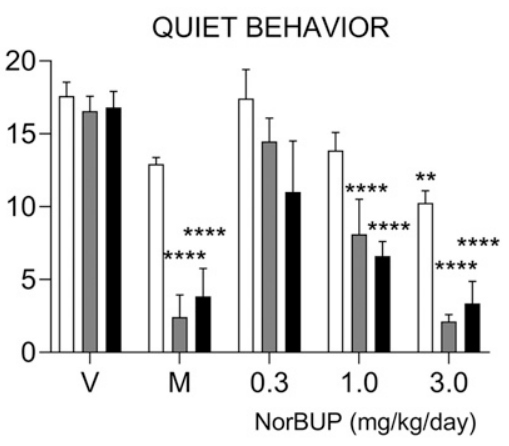

weight gain during prenatal treatment and no evidence of delivery up to 24 days after breeding. However, as shown in Table 3, doses up to and including 3 and $15 \mathrm{mg} / \mathrm{kg}$ per day of NorBUP and morphine, respectively, did not induce spontaneous abortions, or affect litter size $(\mathrm{F}[6,24]=0.8438, P=$ $0.5489)$, pup weight $(\mathrm{F}[5,21]=0.3883, P=0.8512)$, or the number of each sex per litter (males: F[6, 24] $=1.464, P=$ 0.2325 ; females: $\mathrm{F}[6,24]=0.3676, P=0.8922)$, or significantly alter gestational weight gain $(\mathrm{F}[4,72]=1.517, P=$ 0.2063 , pooled slope $=8.455$ g/day, Fig. 4 ).

To assess clinical relevance of the active NorBUP doses and maternal-fetal tissue exposure levels at these doses, we measured concentrations of NorBUP in maternal and fetal blood and brain. We first measured concentrations of NorBUP in maternal whole blood throughout gestation. Following minipump implantation with 1 or $3 \mathrm{mg} / \mathrm{kg}$ per day NorBUP on GD 9, maternal blood concentrations of NorBUP peaked on GD 10, decreased between GDs 10 and 13, plateaued between GDs 13 and 17, and then decreased again between GDs 17 and 20 (Fig. 5A). The median blood concentrations of NorBUP from GD 10 to GD 20 were 1.0 and $9.6 \mathrm{ng} / \mathrm{ml}$ at 1 and $3 \mathrm{mg} / \mathrm{kg}$ per day, respectively. Although there was only a 3 -fold difference in NorBUP dose, blood concentrations were approximately 10-fold higher throughout gestation in dams treated with $3 \mathrm{mg} / \mathrm{kg}$ per day relative to those treated with $1 \mathrm{mg} / \mathrm{kg}$ per day, indicating that NorBUP exhibits nonlinear pharmacokinetics at these doses in the pregnant rat. Maternal blood concentrations of NorBUP in dams treated with $10 \mathrm{mg} / \mathrm{kg}$ per day NorBUP also peaked on GD 10 and decreased on GDs 13 and 17, as observed at the lower doses. However, instead of continuing to decrease, blood concentrations in the $10-\mathrm{mg} / \mathrm{kg}$ per day group increased to peak concentrations on GD 20 (Fig. $5 \mathrm{~A})$. This late-gestation rise in maternal blood concentrations of NorBUP coincided with sudden decreases in maternal weight gain and absence of fetuses on GD 20, which is consistent with the possibility that spontaneous abortion occurred between GDs 17 and 20. One factor that may plausibly lower NorBUP blood concentrations across gestation is maternal weight gain. We corrected NorBUP blood concentrations for weight (Fig. 5B) and compared the slopes of the linear regression lines for the weight-uncorrected and weight-corrected data for each prenatal treatment group using an F-test. There were no differences in these slopes for any group $(1 \mathrm{mg} / \mathrm{kg}$ per day: $\mathrm{F}[1,4]=4.17$, $P=0.11 ; 3 \mathrm{mg} / \mathrm{kg}$ per day: $\mathrm{F}[1,4]=2.764, P=0.17 ; 10 \mathrm{mg} / \mathrm{kg}$ per day: $\mathrm{F}[1,4]=0.4501, P=0.54$ ).

A one-way ANOVA detected group differences in NorBUP blood concentrations on GD 20 in maternal and fetal blood following prenatal treatment with 1 or $3 \mathrm{mg} / \mathrm{kg}$ per day NorBUP $(\mathrm{F}[3,8]=10.86, P=0.0034)$. Sidak's multiple comparison test determined that fetal blood concentrations were significantly 


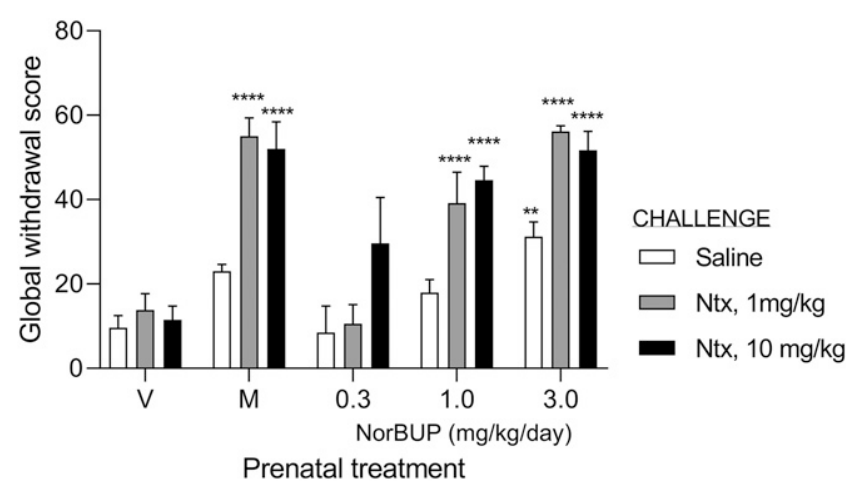

Fig. 2. Global withdrawal scores following prenatal treatment with NorBUP, morphine, or vehicle, and postnatal challenge with naltrexone or saline. Bars represent group means of global withdrawal scores; error bars represent S.E.M. Prenatal treatments are plotted on the $x$-axis and include norbuprenorphine (NorBUP, $0.3-3 \mathrm{mg} / \mathrm{kg}$ per day, s.c.), morphine (M, positive control, $15 \mathrm{mg} / \mathrm{kg}$ per day, s.c.), and vehicle (V, 1:2:1 DMSO/PEG-400/saline, $0.120 \mathrm{ml} /$ day, s.c.). Neonatal pups were challenged with saline $(10 \mathrm{ml} / \mathrm{kg}$, i.p., white bars) or naltrexone (Ntx, $1 \mathrm{mg} / \mathrm{kg}$, i.p., gray bars; Ntx, $10 \mathrm{mg} / \mathrm{kg}$, i.p., black bars) on postnatal day 1 and observed for withdrawal signs. $* * P<0.01$, **** $P<0.0001$ vs. vehicletreated, saline-challenged pups; Dunnett's multiple comparisons test. See Table 2 for detailed statistical results.

greater than maternal blood concentrations following prenatal treatment with $3 \mathrm{mg} / \mathrm{kg}$ per day $\operatorname{NorBUP}(t=4.070, \mathrm{df}=8, P=$ $0.0071)$, but not $1 \mathrm{mg} / \mathrm{kg}$ per day $(t=1.362, \mathrm{df}=8, P=0.3765$; Fig. 6A). Likewise, brain concentrations of NorBUP on GD 20 significantly differed when grouped by origin (i.e., maternal or fetal) and prenatal treatment dose with 1 or $3 \mathrm{mg} / \mathrm{kg}$ per day NorBUP (repeated one-way ANOVA, F[3,6] $=17.27, P=$ 0.0023; matching effectiveness: $\mathrm{F}[2,6]=5.278, P=0.0476)$. Fetal brain concentrations of NorBUP were greater than maternal following prenatal treatment with $3 \mathrm{mg} / \mathrm{kg}$ per day NorBUP $(t=4.312, \mathrm{df}=6, P=0.0100)$, but not $1 \mathrm{mg} / \mathrm{kg}$ per day $(t=2.138$, df $=6, P=0.1469$; Fig. $6 \mathrm{~B})$.

\section{Discussion}

The mechanisms that cause buprenorphine-associated NOWS are poorly understood, but previous reports suggest that the fetus is exposed to NorBUP during prenatal treatment with buprenorphine. Exposure to this full agonist could plausibly contribute to short-term (e.g., NOWS) and long-term (e.g., learning and behavioral problems) adverse effects. The present study is the first to directly test the hypothesis that chronic prenatal exposure to NorBUP can induce physical dependence that leads to NOWS.

We determined that a neonatal behavioral profile consistent with opioid dependence (Barr et al., 1998; Jones and Barr, 2000) was induced in a dose-dependent manner by prenatal treatment with NorBUP. Gestational exposure to NorBUP had no effect on maternal weight gain and neonatal weights, suggesting that the effects of NorBUP on neonatal pups were not caused by disruptions to maternal-fetal nutrition. Altogether, this evidence suggests that the increased withdrawal signs presently observed were directly caused by fetal exposure to NorBUP.

To determine the clinical relevance of the NorBUP doses tested in the model, we measured maternal blood concentrations of NorBUP across gestation to compare them with previously reported NorBUP serum or plasma concentrations

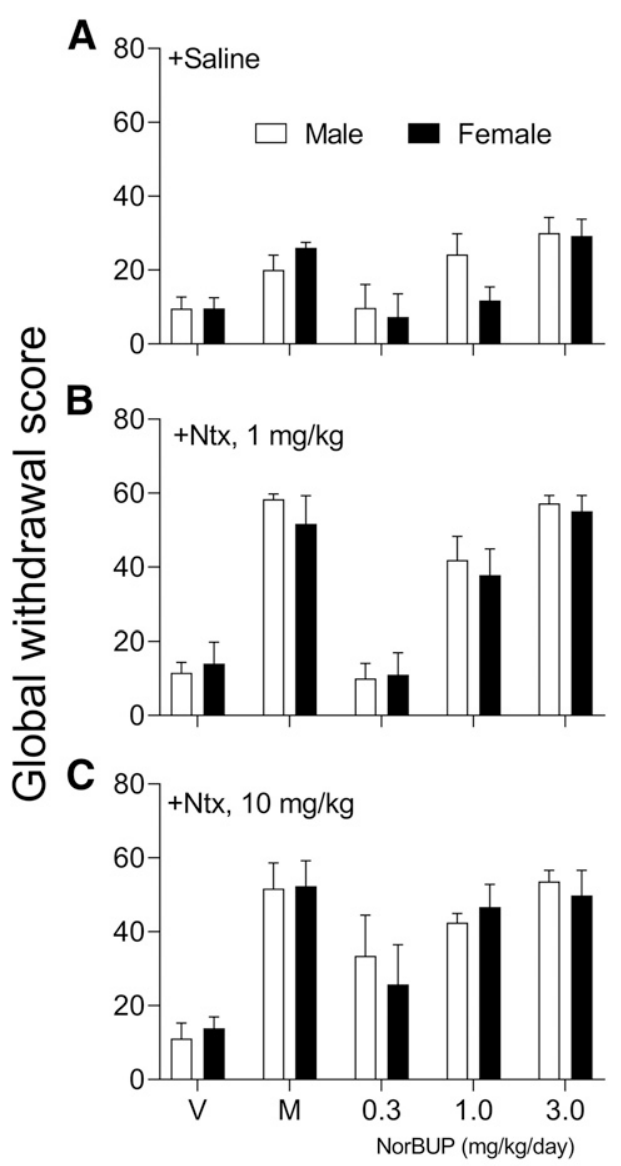

Prenatal treatment

Fig. 3. Sex effects of prenatal treatment on global withdrawal severity. Bars represent the mean global withdrawal score ( $y$-axis) of pups grouped by sex (Male, white bars; Female, black bars) that were postnatally challenged with saline (A), naltrexone, $1 \mathrm{mg} / \mathrm{kg}$ (B), or naltrexone, $10 \mathrm{mg} / \mathrm{kg}$ (C). Prenatal treatments are plotted on the $x$-axis and include norbuprenorphine (NorBUP, $0.3-3 \mathrm{mg} / \mathrm{kg}$ per day, s.c.), morphine (M, positive control, $15 \mathrm{mg} / \mathrm{kg}$ per day, s.c.), and vehicle (V, 1:2:1 DMSO/PEG$400 /$ saline, $0.120 \mathrm{ml} /$ day, s.c.). Error bars represent S.E.M. $P>0.05$, male vs. female for each group; Sidak's multiple comparisons test.

in pregnant women undergoing treatment with buprenorphine. Uncorrected for dose and weight, human serum or plasma concentrations of NorBUP during pregnancy are approximately 1-6 ng/ml (Concheiro et al., 2011; Bartu et al., 2012). Although caution must be taken when comparing plasma, serum, and blood concentrations, as they are not interchangeable, the present study shows that median blood concentrations of NorBUP in the rat model (1.0 and $9.6 \mathrm{ng} / \mathrm{ml}$ at 1 and $3 \mathrm{mg} / \mathrm{kg}$ per day, respectively) are roughly similar to plasma and serum concentrations reported in humans, supporting the clinical and translational relevance of the rat model. Importantly, the blood concentrations of NorBUP in fetal rats in the $1-\mathrm{mg} / \mathrm{kg}$ per day group on GD 20 (mean $=5.1 \mathrm{ng} / \mathrm{ml})$ were within an order of magnitude of those reported in human umbilical cord blood [13.9 ng/ml; (Shah et al., 2016)], serum [1.2 ng/ml; (Bartu et al., 2012)], and plasma $[1.4 \mathrm{ng} / \mathrm{ml}$; (Concheiro et al., 2010)] following prenatal buprenorphine treatment, indicating that fetal exposure is similar in the rat model and human patients.

Some findings related to NorBUP concentrations in maternal blood were unexpected. Steady-state blood concentrations 
TABLE 3

Litter characteristics

\begin{tabular}{|c|c|c|c|c|c|c|c|}
\hline & Vehicle & Morph15 & Morph20 & NorBUP 0.3 & NorBUP 1.0 & NorBUP 3.0 & NorBUP10.0 \\
\hline$N$ & 8 & 3 & 5 & 4 & 7 & 5 & 4 \\
\hline Spont. abortions, \% & 0 & 0 & 40 & 0 & 0 & 0 & $75^{*}$ \\
\hline Litter size, mean (S.D.) ${ }^{a}$ & $10.5(2.5)$ & $11.3(4.0)$ & $9.0(3.6)$ & $13.0(2.6)$ & $11.7(1.4)$ & $11.2(2.4)$ & $11.0(0)$ \\
\hline Males per litter, mean (S.D.) $^{a}$ & $4.8(1.4)$ & $6.0(3.0)$ & $4.3(3.2)$ & $8.2(3.2)$ & $5.7(1.5)$ & $5.8(1.3)$ & $6.0(0)$ \\
\hline Females per litter, mean (S.D. $)^{a}$ & $5.8(1.7)$ & $5.3(1.1)$ & $4.7(1.5)$ & $4.8(2.1)$ & $6.0(2.0)$ & $5.4(1.5)$ & $5.0(0)$ \\
\hline Neonatal pup weights, g, mean (S.D.) & $5.9(0.5)^{b}$ & $5.7(0.3)$ & NA & $5.9(0.5)$ & $5.8(0.4)$ & $5.6(0.3)$ & $5.7(0)$ \\
\hline
\end{tabular}

$* P=0.018$, vs. vehicle (Fisher's exact test).

${ }^{a}$ Includes data from dams that delivered ( $n=3$ and 1 for Morph-20 and NorBUP-10, respectively).

${ }^{b_{n}}=7$, NA, not available.

were expected to be reached well within 24 hours of infusion initiation, on the basis of previous reports of NorBUP having an elimination half-life in nonpregnant rats of approximately 1 hour (Ohtani et al., 1994) or less (Yassen et al., 2007) following intravenous administration. However, blood concentrations peaked on GD 10, 24 hours after implantation of primed minipumps, and decreased to steady-state concentrations by GD 13, indicating that steady state was not reached until 24-96 hours after the infusion began. Although the unexpected delay to steady state could have been caused by differences between studies in route of administration, or rat sex and strain, it was probably the result of the normal physiologic changes that occur during pregnancy. These changes include the addition of the placental and fetal compartments, increased renal and hepatic activity, and increased plasma volume, which lowers drug concentrations in the blood by a dilution effect. Altogether, these physiologic changes tend to increase drug clearance and volume of distribution, leading to decreased net blood concentrations of drugs and metabolites (Schoonover and Littell, 2001). Weight gain across gestation can decrease steady state blood levels; however, weight gain alone did not explain the transgestational changes in steady-state blood levels. The rebound of NorBUP to peak levels that coincided with spontaneous abortions in the $10-\mathrm{mg} / \mathrm{kg}$ per day group further supports the notion that the physiologic changes associated with pregnancy decreased NorBUP levels. Likewise, human studies have shown that plasma concentrations of NorBUP tend to decrease with advancing gestation (Concheiro et al., 2011), further supporting the translational relevance of the rat model.

The transplacental passage of NorBUP is not well understood, but maternal serum concentrations of NorBUP have been shown to positively and linearly predict NorBUP concentrations in umbilical cord serum in humans (Bartu et al., 2012). The close relationship between maternal and umbilical cord (i.e., fetal) serum concentrations of NorBUP suggests that NorBUP measured in fetal tissues probably crossed the placenta instead of being formed by fetal metabolism of buprenorphine. In the present study, NorBUP concentrations were two to four times higher in fetal versus maternal blood and brain tissues on GD 20 (Fig. 6). The new data provided by the current study strongly suggests that NorBUP is sequestered in the fetus late in gestation.

In the present study, tripling NorBUP dose from 1 to $3 \mathrm{mg} / \mathrm{kg}$ per day increased maternal blood concentrations of NorBUP by approximately 10 -fold, suggesting that NorBUP exhibits nonlinear pharmacokinetics at these doses. These findings are consistent with a previous report of nonlinear pharmacokinetics of NorBUP in nonpregnant rats following a single 20-minute intravenous infusion (Yassen et al., 2007). The new finding is important considering that fetal NorBUP exposure increases withdrawal severity in a dose-dependent manner, and because fetal NorBUP exposure is linearly predicted by maternal circulatory levels (Bartu, et al., 2012). This altogether suggests that even small increases in maternal NorBUP dose may amplify fetal NorBUP exposure and negative effects on the fetus. In the present study, the nonlinear increase in maternal blood concentrations was constant throughout gestation, suggesting that nonlinear pharmacokinetics are maintained throughout gestation despite physiologic changes of pregnancy that tend to enhance drug elimination. In contrast, another study that measured NorBUP blood concentrations over time following administration of buprenorphine in nonpregnant rats reported that NorBUP exhibited linear pharmacokinetics (Gopal et al., 2002), suggesting that the pharmacokinetics of NorBUP may be affected by its formation from and interactions with buprenorphine. Investigations of interactions between NorBUP and its parent drug in the maternal-fetal dyad are needed to better understand the role of NorBUP in the development of fetal opioid dependence following chronic treatment with buprenorphine.

The existence of sex differences in NOWS is controversial. After controlling for maternal-neonatal variables that may influence NOWS, some human studies reported that male

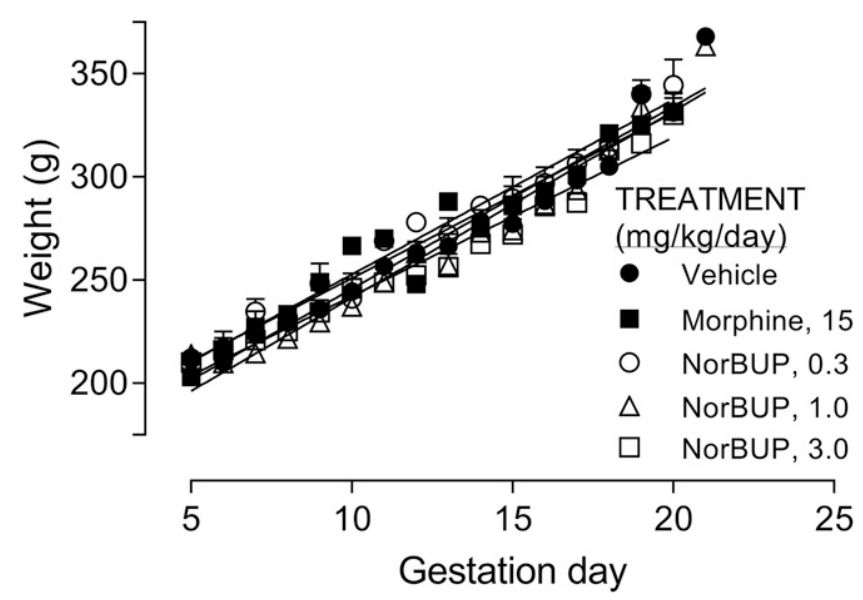

Fig. 4. Maternal weight gain during prenatal treatment. Data are grouped by prenatal treatment (vehicle, closed circles; morphine, $15 \mathrm{mg} / \mathrm{kg}$ per day, closed squares; NorBUP, $0.3 \mathrm{mg} / \mathrm{kg}$ per day, open circles; NorBUP, $1 \mathrm{mg} / \mathrm{kg}$ per day, open triangles; NorBUP, $3 \mathrm{mg} / \mathrm{kg}$ per day, open squares). Data points represent group mean weights ( $y$-axis) plotted by gestation day ( $x$-axis); error bars represent S.E.M. Each line represents the linear regression analysis best-fit of the data points for each prenatal treatment group. There were no group differences in the slopes of the linear regressions $(\mathrm{F}[4,72]=1.517, P=0.2063$, pooled slope $=8.455 \mathrm{~g} / \mathrm{day})$. 

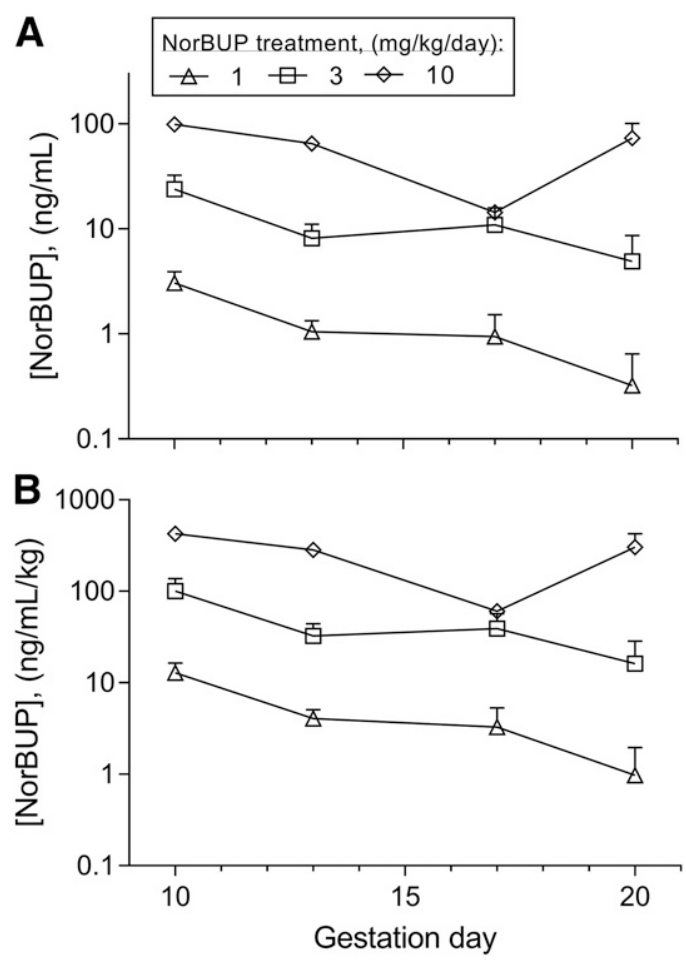

Fig. 5. Maternal blood concentrations of NorBUP in NorBUP-treated dams. Data are grouped by prenatal NorBUP dose $(1 \mathrm{mg} / \mathrm{kg}$ per day, open triangles; $3 \mathrm{mg} / \mathrm{kg}$ per day, open squares; $10 \mathrm{mg} / \mathrm{kg}$ per day, open diamonds), and data points represent mean group blood concentrations of NorBUP ( $y$-axis) uncorrected (A) and corrected (B) for body weight and plotted by gestation day ( $x$-axis). Error bars represent S.E.M. Blood concentrations peaked approximately 24 hours after minipump implantation and decreased throughout gestation in a weight-independent manner. NorBUP blood concentrations measured in the $10 \mathrm{mg} / \mathrm{kg}$ per day group $(n=2)$ increased between GDs 17 and 20 following suspected spontaneous abortions. Transgestational weight changes did not significantly affect transgestational changes in blood concentrations $(n=3$ for 1 and $3 \mathrm{mg} / \mathrm{kg}$ per day prenatal treatment groups).

neonates are slightly more likely than females to be diagnosed with NOWS (O'Connor et al., 2013; Charles et al., 2017), experience more severe withdrawal signs (Jansson et al., 2007, 2010; O'Connor et al., 2013), require longer hospital stays and treatment duration (Jansson et al., 2007, 2010), and more probably would require pharmacological treatment (Jansson et al., 2010; O'Connor et al., 2013), whereas other human studies reported no sex effect of these NOWS endpoints (Holbrook and Kaltenbach, 2010; Unger et al., 2011). With the exception of stress caused by shipping and minipump implantation surgeries, variables in the present study in rats were highly controlled relative to human studies. These variables include opioid exposure, diet, housing conditions, and interindividual differences and are therefore well-powered to detect differences in NOWS owing to sex. We detected no sex differences in withdrawal severity following gestational exposure to morphine or NorBUP at a low dose $(0.3 \mathrm{mg} / \mathrm{kg}$ per day) or high dose ( $3 \mathrm{mg} / \mathrm{kg}$ per day). Furthermore, no opioid treatment affected the proportion of males versus females in each litter, indicating that opioid exposure did not cause sexspecific resorptions. The discrepancies in the reports of sex effects may be specific to the type of opioid exposure and/or the species in the study. More studies in humans and rodent models of NOWS are needed to confirm and understand sex differences in NOWS.
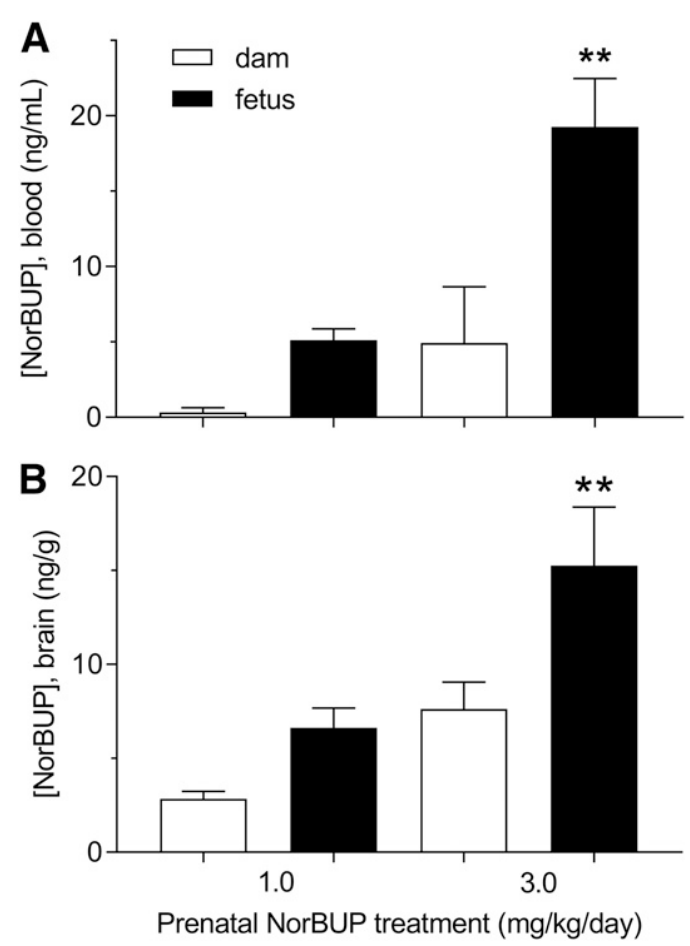

Fig. 6. Sequestration of NorBUP in fetal vs. maternal tissues following prenatal NorBUP treatment. Bars represent the mean NorBUP concentrations in blood (A) and brain (B) harvested from dams (white bars) and fetuses (black bars) on gestation day 20. Error bars represent S.E.M. Bars are grouped by prenatal treatment ( 1 or $3 \mathrm{mg} / \mathrm{kg}$ per day NorBUP). NorBUP concentrations were significantly higher in the blood and brain of fetuses relative to maternal blood and brain in the $3 \mathrm{mg} / \mathrm{kg}$ per day NorBUP group **P $<0.01$, Sidak's multiple comparisons test.

In conclusion, the present study is the first to provide proof of the concept that NorBUP can induce fetal opioid dependence and lead to NOWS. This finding is significant for two reasons. First, it provides the first evidence supporting a novel mechanistic framework to explain the lack of dose-dependent relationship between maternal buprenorphine dose and neonatal withdrawal severity. The metabolism of buprenorphine to NorBUP and the distribution of NorBUP to the fetus are predominately controlled by select cytochrome P450s (Picard et al., 2005; Chang et al., 2006) and may be modulated by placental P-glycoprotein expression and activity (Alhaddad et al., 2012; Brown et al., 2012), respectively. The expression and activity of these highly polymorphic enzymes vary considerably across the population (Hemauer et al., 2010; Samer et al., 2010; Klein and Zanger, 2013), and thus may mediate the lack of dose dependence between maternal buprenorphine and NOWS by influencing fetal exposure to NorBUP. Second, this study suggests for the first time that decreasing fetal exposure to NorBUP may diminish NOWS. These findings highlight novel strategies for improving treatment of OUD during pregnancy by decreasing NOWS liability. These potential strategies, which require further research and development, include identifying maternal-fetal dyads with genetic variants of cytochrome $\mathrm{P} 450$ and P-glycoprotein that promote fetal exposure to NorBUP, developing new medications that selectively upregulate placental P-glycoprotein to efflux NorBUP from the fetal-placental compartment, and modifying buprenorphine to resist metabolism to NorBUP. 


\section{Acknowledgments}

We acknowledge the NIDA Drug Supply Program for providing NorBUP for this study.

\section{Authorship Contributions}

Participated in research design: Russell, Alund, Owens, Fantegrossi, Moran, Brents.

Conducted experiments: Griffin, Caperton, Russell, Cabanlong,

Wilson, Urquhart, Martins, Zita, Alund, Brents.

Contributed new reagents or analytic tools: Patton, Moran.

Performed data analysis: Griffin, Brents.

Wrote or contributed to the writing of the manuscript: Griffin, Caperton, Russell, Cabanlong, Wilson, Urquhart, Martins, Zita, Patton, Alund, Owens, Fantegrossi, Moran, Brents.

\section{References}

Alhaddad H, Cisternino S, Declèves X, Tournier N, Schlatter J, Chiadmi F, Risède P, Smirnova M, Besengez C, Scherrmann JM, et al. (2012) Respiratory toxicity of buprenorphine results from the blockage of P-glycoprotein-mediated efflux of norbuprenorphine at the blood-brain barrier in mice. Crit Care Med 40:3215-3223.

Barr GA, Zmitrovich A, Hamowy AS, Liu PY, Wang S, and Hutchings DE (1998) Neonatal withdrawal following pre- and postnatal exposure to methadone in the rat. Pharmacol Biochem Behav 60:97-104.

Bartu AE, Ilett KF, Hackett LP, Doherty DA, and Hamilton D (2012) Buprenorphine exposure in infants of opioid-dependent mothers at birth. Aust $N Z \mathrm{~J}$ Obstet Gynaecol 52:342-347.

Brogly SB, Saia KA, Walley AY, Du HM, and Sebastiani P (2014) Prenatal buprenorphine versus methadone exposure and neonatal outcomes: systematic review and meta-analysis. Am J Epidemiol 180:673-686.

Brown SM, Campbell SD, Crafford A, Regina KJ, Holtzman MJ, and Kharasch ED (2012) P-glycoprotein is a major determinant of norbuprenorphine brain exposure and antinociception. J Pharmacol Exp Ther 343:53-61.

Chang Y, Moody DE, and McCance-Katz EF (2006) Novel metabolites of buprenorphine detected in human liver microsomes and human urine. Drug Metab Dispos 34: $440-448$.

Charles MK, Cooper WO, Jansson LM, Dudley J, Slaughter JC, and Patrick SW (2017) Male sex associated with increased risk of neonatal abstinence syndrome. Hosp Pediatr 7:328-334.

Concheiro M, Jones HE, Johnson RE, Choo R, and Huestis MA (2011) Preliminary buprenorphine sublingual tablet pharmacokinetic data in plasma, oral fluid, and sweat during treatment of opioid-dependent pregnant women. Ther Drug Monit 33: 619-626.

Concheiro M, Jones HE, Johnson RE, Choo R, Shakleya DM, and Huestis MA (2010) Umbilical cord monitoring of in utero drug exposure to buprenorphine and correlation with maternal dose and neonatal outcomes. J Anal Toxicol 34:498-505.

Gopal S, Tzeng TB, and Cowan A (2002) Characterization of the pharmacokinetics of buprenorphine and norbuprenorphine in rats after intravenous bolus administration of buprenorphine. Eur J Pharm Sci 15:287-293.

Hemauer SJ, Nanovskaya TN, Abdel-Rahman SZ, Patrikeeva SL, Hankins GD, and Ahmed MS (2010) Modulation of human placental P-glycoprotein expression and activity by MDR1 gene polymorphisms. Biochem Pharmacol 79:921-925.

Holbrook A and Kaltenbach K (2010) Gender and NAS: does sex matter? Drug Alcohol Depend 112:156-159.

Huang P, Kehner GB, Cowan A, and Liu-Chen LY (2001) Comparison of pharmacological activities of buprenorphine and norbuprenorphine: norbuprenorphine is a potent opioid agonist. J Pharmacol Exp Ther 297:688-695.

Jansson LM, Dipietro JA, Elko A, and Velez M (2010) Infant autonomic functioning and neonatal abstinence syndrome. Drug Alcohol Depend 109:198-204.
Jansson LM, Dipietro JA, Elko A, and Velez M (2007) Maternal vagal tone change in response to methadone is associated with neonatal abstinence syndrome severity in exposed neonates. J Matern Fetal Neonatal Med 20:677-685.

Jansson LM, Velez M, and Harrow C (2009) The opioid-exposed newborn: assessment and pharmacologic management. J Opioid Manag 5:47-55.

Jones HE, Dengler E, Garrison A, O'Grady KE, Seashore C, Horton E, Andringa K, Jansson LM, and Thorp J (2014) Neonatal outcomes and their relationship to maternal buprenorphine dose during pregnancy. Drug Alcohol Depend 134: 414-417.

Jones HE, Kaltenbach K, Heil SH, Stine SM, Coyle MG, Arria AM, O'Grady KE, Selby P, Martin PR, and Fischer G (2010) Neonatal abstinence syndrome after methadone or buprenorphine exposure. N Engl J Med 363:2320-2331.

Jones KL and Barr GA (2000) Opiate withdrawal in the fetal rat: a behavioral profile. Pharmacol Biochem Behav 66:419-424.

Klein K and Zanger UM (2013) Pharmacogenomics of cytochrome P450 3A4: recent progress toward the "missing heritability" problem. Front Genet 4:12.

O'Connor AB, O'Brien L, and Alto WA (2013) Are there gender related differences in neonatal abstinence syndrome following exposure to buprenorphine during pregnancy? J Perinat Med 41:621-623.

O'Connor AB, O'Brien L, and Alto WA (2016) Maternal buprenorphine dose at delivery and its relationship to neonatal outcomes. Eur Addict Res 22:127-130.

Ohtani M, Kotaki H, Uchino K, Sawada Y, and Iga T (1994) Pharmacokinetic analysis of enterohepatic circulation of buprenorphine and its active metabolite, norbuprenorphine, in rats. Drug Metab Dispos 22:2-7.

Patrick SW, Davis MM, Lehmann CU, and Cooper WO (2015) Increasing incidence and geographic distribution of neonatal abstinence syndrome: United States 2009 to 2012 [published correction appears in J Perinatol (2015) 35:667]. J Perinatol 35:650-655.

Patrick SW, Schumacher RE, Benneyworth BD, Krans EE, McAllister JM, and Davis MM (2012) Neonatal abstinence syndrome and associated health care expenditures: United States, 2000-2009. JAMA 307:1934-1940.

Picard N, Cresteil T, Djebli N, and Marquet P (2005) In vitro metabolism study of buprenorphine: evidence for new metabolic pathways. Drug Metab Dispos 33: $689-695$.

Samer CF, Daali Y, Wagner M, Hopfgartner G, Eap CB, Rebsamen MC, Rossier MF Hochstrasser D, Dayer P, and Desmeules JA (2010) Genetic polymorphisms and drug interactions modulating CYP2D6 and CYP3A activities have a major effect on oxycodone analgesic efficacy and safety. Br J Pharmacol 160:919-930.

Schoonover L and Littell C (2001) Pharmacokinetics of drugs during pregnancy and lactation, in Drug Therapy in Pregnancy (Yankowitz J and Niebyl J eds), 3rd ed, pp 5-17, Lippincott Williams and Wilkins, Philadelphia.

Shah D, Brown S, Hagemeier N, Zheng S, Kyle A, Pryor J, Dankhara N, and Singh P (2016) Predictors of neonatal abstinence syndrome in buprenorphine exposed newborn: can cord blood buprenorphine metabolite levels help? Springerplus 5:854.

Unger A, Jagsch R, Bäwert A, Winklbaur B, Rohrmeister K, Martin PR, Coyle M, and Fischer G (2011) Are male neonates more vulnerable to neonatal abstinence syndrome than female neonates? Gend Med 8:355-364.

Yassen A, Kan J, Olofsen E, Suidgeest E, Dahan A, and Danhof M (2007) Pharmacokinetic-pharmacodynamic modeling of the respiratory depressant effect of norbuprenorphine in rats. J Pharmacol Exp Ther 321:598-607.

Zedler BK, Mann AL, Kim MM, Amick HR, Joyce AR, Murrelle EL, and Jones HE (2016) Buprenorphine compared with methadone to treat pregnant women with opioid use disorder: a systematic review and meta-analysis of safety in the mother, fetus and child. Addiction 111:2115-2128.

Zhu H and Barr GA (2004) The role of AMPA and metabotropic glutamate receptors on morphine withdrawal in infant rats. Int J Dev Neurosci 22:379-395.

Address correspondence to: Dr. Lisa K. Brents, Department of Pharmacology and Toxicology, University of Arkansas for Medical Sciences, 4301 West Markham Street, Slot 611, Little Rock, AR 72205. E-mail: LBRENTS@ uams.edu 